\title{
The utilization of light weight boards for reducing air emissions by the German wood industry - a perspective?
}

\section{Der Einsatz von Leichtbauplatten zur Senkung von Luftemissionen aus der deutschen Holzindustrie - eine Perspektive?}

Silke Feifel ${ }^{1 *}$, Witold-Roger Poganietz ${ }^{1}$ and Liselotte Schebek ${ }^{2}$

\begin{abstract}
Background: Resource saving product development is one competitive advantage for companies. Forestbased industries try to realize this amongst others with lightweight board solutions.

Using lightweight boards is discussed in the wood industry for several years and could be technically realized nowadays on an industrial scale. Advantages by using lightweight boards are expected in costs due to reduced wood requirements as well as in respect to logistics. In discussion are potential environmental advantages of an enhanced use of lightweight boards.

The objective of the paper is to identify and quantify the environmental impacts due to an increased use of lightweight boards in the furniture industry. The focus rests on the so-called Kyoto greenhouse gas emissions, NMVOC and formaldehyde.
\end{abstract}

Results: The study grounds on a process-based material flow model, which depicts the use of forest resources in Germany. The situation in 2005 is compared with a scenario, which describes a partial substitution of conventional boards by lightweight boards using sandwich design.

Overall the greenhouse gas emissions will decrease, even though the overall decline is rather small.

Conclusions: By this lightweight boards could be a perspective for the German wood-industry to reduce emissions into air noteworthy. The main causes are a reduced requirement for adhesives and binders, logistics and thermal use of residuals.

Keywords: Light weight boards, Material flow analysis, Greenhouse gas emissions, Environmental performance

\footnotetext{
* Correspondence: silke.feifel@kit.edu

${ }^{1}$ Karlsruher Institut für Technologie, Institut für Technikfolgenabschätzung

und Systemanalyse, Hermann-von-Helmholtz-Platz 1, D-76344

Eggenstein-Leopoldshafen, Germany

Full list of author information is available at the end of the article
} 


\section{Zusammenfassung}

Background: Ressourcenschonende Produktentwicklungen sind ein entscheidender Wettbewerbsvorteil für Unternehmen. In der Holzindustrie wird dies u.a. mit Leichtbauplatten verfolgt.

Der Einsatz leichter Holzwerkstoffplatten wird seit Jahren in der Holzindustrie diskutiert und ist heute technisch ausgereift. Erwartet werden unternehmensabhängig Kostenvorteile durch einen geringeren Holzeinsatz, aber auch Vorteile in der Logistik. Offen ist, welche ökologischen Konsequenzen, ggf. Vorteile mit dem Einsatz von Leichtbauplatten verbunden sind.

Das Ziel des Beitrags ist die Identifikation und die Quantifizierung der ökologischen Wirkungen eines verstärkten Einsatzes von Leichtbauplatten in der Holzindustrie. Hierbei konzentriert sich der Beitrag auf die im Kioto-Protokoll reglementierten Treibhausgase, sowie NMVOCs und Formaldehyd.

Results: Für die Analyse wird mit Hilfe eines prozessbasierten Materialflussmodells, welches die Nutzung forstlicher Ressourcen in Deutschland abbildet, die Situation in 2005 als Referenz mit einem Szenario verglichen, in dem Spanplatten partiell durch Leichtbauplatten in Sandwichbauweise substituiert werden.

Im Ergebnis sinken die Treibhausgasemissionen durchgängig, wenn die Minderungen auch gering ausfallen.

Conclusions: Damit stellen leichte Holzwerkstoffplatten für die deutsche Holzindustrie eine Perspektive, ihre Luftemissionen zu senken. Die Ursachen für die Senkungen sind zu wesentlichen Teilen im Bereich der Klebstoffe und Bindemittel, der Logistik und der thermischen Nutzung von Produktionsresten zu finden.

Schlagwörter: Leichtbauplatten, Materialflussanalyse, Treibhausgasemissionen, Umweltwirkungen

\section{Einleitung}

Ressourcenschonende Produktentwicklungen sind wichtige Innovationen, um die Wettbewerbsfähigkeit und die Zukunftsfähigkeit von Unternehmen und ganzen Branchen zu sichern. Dies wird in der Holzindustrie u. a. mit Leichtbauplatten verfolgt.

Der Einsatz von Leichtbauplatten in der Möbelfertigung und anderen Anwendungsfeldern wie bspw. dem Innenausbau als Substitut konventioneller Holzwerkstoffplatten ist ein in Fachkreisen viel diskutiertes Thema [1-3]. Die notwendige Technik zur Verarbeitung der Platten hat inzwischen Marktreife erreicht [4]. Am Markt verfügbar sind verschiedene Bauformen leichter Platten wie Sandwichbauweisen mit Papiermittellage, z.B. sogenannte Expansionswabenplatten, oder homogene Werkstoffe aus der Gruppe der Mitteldichten Faserplatten (MDF) wie z.B. die Ultraleicht-MDF [5]. Die Vorteile von Leichtbauplatten liegen - bedingt durch das Gewicht der Platten - in der Logistik und, abhängig von der Bauform, auch in der Umweltperformance des Produktionsprozesses der Platten im Vergleich zu denen von konventionellen Holzwerkstoffen. Bezüglich der Umweltperformance können dabei zwei Gruppen unterschieden werden, deren ökologische Wirkungen geringer (Sandwichplatten mit Papiermittellage) sowie größer (Sandwichplatten mit Kunststoff- oder Vollholzmittellagen) im Vergleich zu den konventionellen Spanplatten und MDF sind [6,7].

Die wenigen Arbeiten zu Leichtbauplatten [8-10] fokussieren auf die Analyse vor allem technischer Eigenschaften von mehr oder weniger umfassend beschriebenen Produktsystemen. Allen Untersuchungen gemein ist aber das Fehlen der Interdependenzen zwischen konkurrierenden Prozesspfaden. Beispielsweise kann ein verstärkter Einsatz von Leichtbauplatten ein sinkendes Angebot an Industrierestholz bedeuten und damit möglicherweise ein verstärkter Einsatz von fossilen Brennstoffen als Substitut von Industrierestholz. Ebenso kann es zu einer Limitierung der stofflichen Nutzung von Industrierestholz kommen und damit einhergehend ein zusätzlicher Bedarf an Rohholz, das zu einem verstärkten Holzeinschlag führen wird. Eine Hochrechnung der ökologischen Vorteile aus den Ergebnissen von Produktökobilanzen für die gesamte Holzindustrie ist aufgrund der vielfältigen Änderungen im Produktionsablauf bedingt durch Leichtbauplatten und den dadurch generierten Substitutions- und Rückkopplungseffekten nicht möglich. Daher muss für die Betrachtung der Umweltrelevanz des Einsatzes von Leichtbauplatten in der Möbelfertigung der Systemrahmen über das eigentliche Produkt Leichtbauplatte und die Möbelfertigung deutlich hinausgehen und die Zusammenhänge in der Holzindustrie mit den wesentlichen vor- und gelagerten Prozessen erfassen.

Das Ziel der Arbeit ist die Identifikation und Quantifizierung der Emissionen der gesamten Holzindustrie durch einen verstärkten Einsatz von Leichtbauplatten, wobei als Grundlage ein prozessbasiertes Materialflussmodell der Holzindustrie mit den wesentlichen vor- und nachgelagerten Prozessen verwendet wird. Da Spanplatten sowie Leichtbauplatten primär in der Möbelindustrie eingesetzt werden [1], fokussieren die 
folgenden Ausführungen auf dieses Segment der Holzindustrie, trotz der weiteren vielfältigen Einsatzmöglichkeiten beider Plattenarten [3].

Aufgrund der aktuellen politischen und ökologischen Relevanz des Klimawandels fokussiert der Beitrag auf die treibhauswirksamen Emissionen (THG), wie sie nach dem Kioto-Protokoll festgelegt sind und im Ausstoß reglementiert wurden, sofern sie in der Holzindustrie ausgestoßen werden. Daneben werden die Folgen eines Einsatzes von Leichtbauplatten auf die Emission von flüchtigen organischen Verbindungen ohne Methan (Non-methane Volatile Organic Compounds; NMVOC) und Formaldehyd $\left(\mathrm{CH}_{2} \mathrm{O}\right)$ analysiert. NMVOC sind stets von Interesse, wenn Lackiervorgänge in die Betrachtungen eingehen und Formaldehyd ist eine im Zusammenhang mit Holzwerkstoffen mehr aus vergangenen Erfahrungen heraus öffentlich diskutierte und für die menschliche Gesundheit relevante Größe. Die gewonnenen Erkenntnisse werden dazu genutzt, die Ursachen der veränderten Emissionsmengen und -struktur $\mathrm{zu}$ identifizieren und zu plausibilisieren. Weiterhin erfolgt eine Einordnung des Emissionsminderungspotenzials zu den Gesamtemissionen der deutschen Holzindustrie.

In dem nachfolgenden Kapitel 2 wird zunächst die Leichtbauplatte mit der konventionellen Spanplatte verglichen und Anpassungsnotwendigkeiten in der Möbelindustrie beschrieben. Kapitel 3 zielt auf die Darstellung der Vorgehensweise. Konkret wird zunächst die methodische Grundlage skizziert, um dann das Referenzsystem sowie das Analyseszenario zu thematisieren. Die Ergebnisse werden in Kapitel 4 vorgestellt und in Kapitel 5 diskutiert. Im Abschlusskapitel 6 erfolgt ein Fazit.

\section{Die Leichtbauplatte im Vergleich zur Spanplatte}

Spanplatten sind plattenförmige Holzwerkstoffe, die durch Verpressen von kleinen und kleinsten Holzteilen und/oder anderen holzartigen Faserstoffen wie z.B. Stroh mit Klebstoffen und Bindemitteln hergestellt werden (s. Abbildung 1). Spanplatten werden überwiegend als Flachpressplatten insbesondere für die Möbelfertigung in ein- oder mehrschichtigem Aufbau produziert. Die Platteneigenschaften hängen in hohem Maß von der Rohdichte der Platten, aber auch von den verwendeten Bindemitteln ab [11]. Die Rohdichten dieser Platten liegen bei $600-750 \mathrm{~kg} / \mathrm{m}^{3}[5]$.

Leichtbauplatten sind in verschiedenen Bauformen am Markt erhältlich. Für die Möbelfertigung sind $z$. Zt. primär Wabenplatten interessant. Wabenplatten sind Plattenwerkstoffe, die in Sandwichbauweise zusammengesetzt sind und deren Mittellagen aus senkrecht zur Fläche stehenden, dünnwandigen Hohlkammerprofilen aus Papier bestehen [5]. Wabenplatten unterscheiden sich in den Materialien und den Zellweiten der Waben. Zum einen

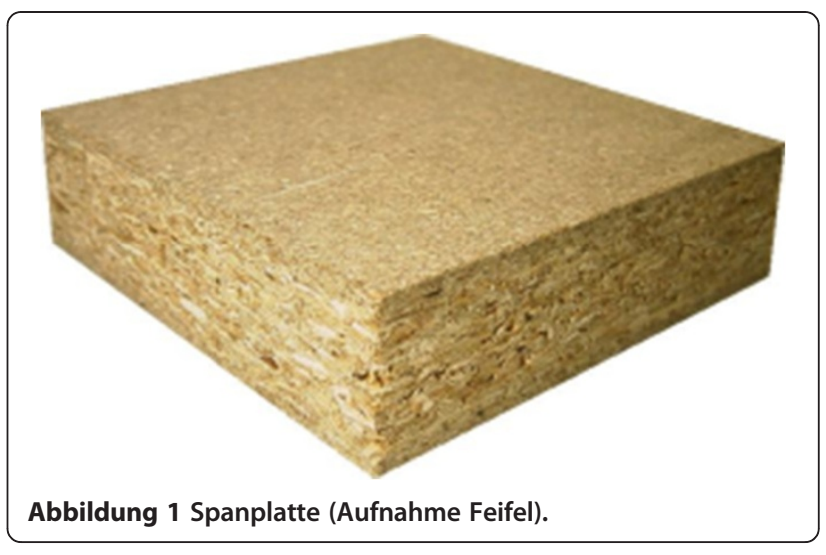

werden unterschiedliche Deckschichten verarbeitet, z.B. Dünnspan, Sperrholz, Hartfaser, MDF roh und lackiert bzw. beschichtet, Furnier oder Laminat (s. Abbildung 2). Zum anderen sind die Wabenstrukturen unterschiedlich dicht und können aus unterschiedlichen Materialien wie $\mathrm{z}$. B. Papier, Kunststoff oder Metall gefertigt werden. Die Rohdichten dieser Werkstoffe betragen, abhängig von Plattendicke und Materialeinsatz für Mittellage und Deckschicht, zwischen $100-450 \mathrm{~kg} / \mathrm{m}^{3}$ [5].

Die Produktion von Leichtbauplatten wird in der folgenden Analyse durch den Materialmix dünner Spanplatten (Deckschichten), Papier (Mittellage) und Bindemitteln abgebildet und der konventionellen Spanplatte hinsichtlich Material- und Energieinput gegenüber gestellt (s. Tabelle 1).

Die Nutzung von Sandwichplatten in der Möbelfertigung bedingt - im Vergleich zur Nutzung von Span- und Faserplatten - eine andersartige Befestigung von Beschlägen und Handhabung der Schmalkanten. Beim Einsatz von Wabenplatten ist eine Modifizierung der Produktionsanlagen unumgänglich. In der Untersuchung wird dies durch die Anpassung einzelner Prozessschritte in der Möbelfertigung realisiert. Es ergeben sich zwei zusätzliche Arbeitsschritte (s. Abbildung 3), die sich jedoch, in Abhängigkeit vom Fertigungsprinzip, auch an anderer Stelle der Prozesskette einfügen können:

- Das Vorbereiten der Kanten durch Einbringen von Stützkanten, um ein Einsacken der z.B. AcrylnitrilButadien-Styrol -Dekorkante (ABS) aufgrund der Hohlbauweise zu verhindern [13].

- Die Vorbereitung zum Setzen der Beschläge durch Klebedübel [14] oder Klebemuffen [15] und Klebstoffeinbringung wie z.B. Polyurethan (PUR).

Ausgehend von qualitativ gleichwertigen Möbeln, kann nach bisherigen Erfahrungen davon ausgegangen werden, dass die technische Lebensdauer der unterschiedlichen 

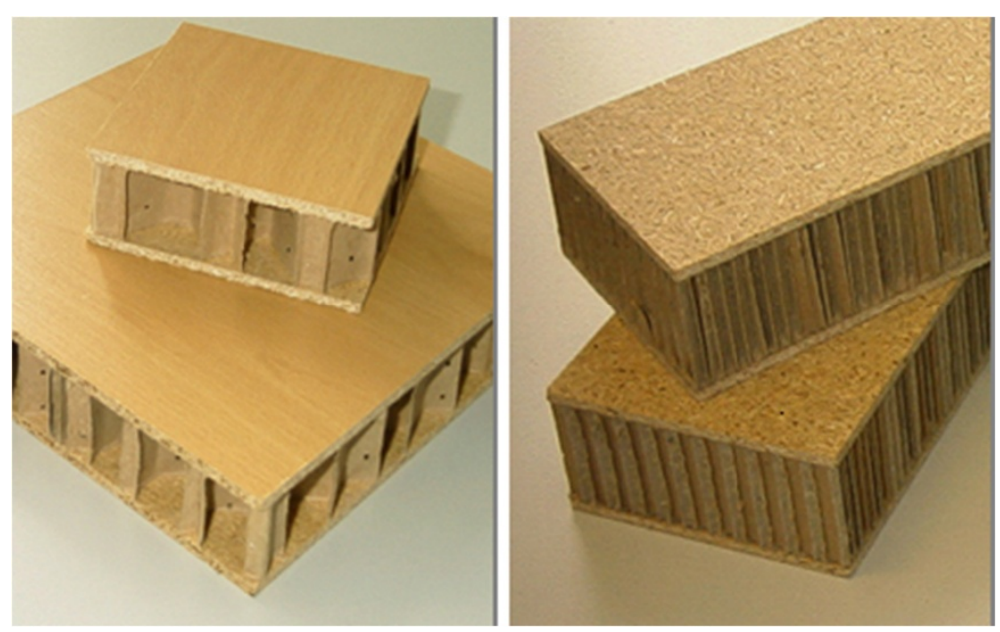

Abbildung 2 Leichtbauplatten: Expansionswaben--Mittellage mit Dünn--Spandeckschichten, Oberfläche, beschichtet (links) und Wellstegmittellage mit Dünn--Spandeckschichten, Oberfläche roh (rechts) (Aufnahme Feifel).

Bauformen gleich ist, d.h. es wird hinsichtlich der Nutzungsdauer der Möbel keine Unterscheidung getroffen.

Die oben beschriebenen Unterschiede in der Herstellung von Leichtbauplatten im Vergleich zur konventionellen Spanplatte und die Anpassungsnotwendigkeiten in der Möbelindustrie werden für die Analyse in ein prozessbasiertes Materialflussmodell der Forst-HolzNutzungskette implementiert, welches im Folgenden vorgestellt wird.

\section{Vorgehensweise}

\section{Methodische Grundlage}

Die Grundlage der Analyse bildet ein Materialflussmodell, welches für 2005 die Nutzung forstlicher Ressourcen in Deutschland auf der Ebene von Produktionsprozessen umfassend darstellt. Zusätzlich werden die relevanten Vorketten erfasst. Für das Jahr 2005 wird unterstellt, dass keine Leichtbauplatten in der Holzindustrie eingesetzt werden. Diese Situation wird mit einem Szenario verglichen, in dem Spanplatten partiell durch Leichtbauplatten ersetzt werden.

Tabelle 1 Gegenüberstellung der Material- und Energieinputs für Leichtbau- und Spanplatte

\begin{tabular}{|c|c|c|}
\hline & Leichtbauplatten [6] & Spanplatten [12] \\
\hline Industrierestholz $\left[\mathrm{kg} / \mathrm{m}^{3}\right]$ & 72,8 & 254,7 \\
\hline Industrieholz $\left[\mathrm{m}^{3} / \mathrm{m}^{3}\right]$ & 0,1 & 0,5 \\
\hline Bindemittel $\left[\mathrm{kg} / \mathrm{m}^{3}\right]$ & 34,0 & 61,6 \\
\hline Papier $\left[\mathrm{kg} / \mathrm{m}^{3}\right]$ & 5,5 & 0,0 \\
\hline Energie $\left[\mathrm{kWh} / \mathrm{m}^{3}\right]$ & 471,7 & 874,0 \\
\hline $\begin{array}{l}\text { davon: Energie thermisch } \\
{\left[\mathrm{kWh} / \mathrm{m}^{3}\right]}\end{array}$ & 380,2 & 643,0 \\
\hline Energie elektrisch $\left[\mathrm{kWh} / \mathrm{m}^{3}\right]$ & 91,5 & 231,0 \\
\hline
\end{tabular}

Die methodische Grundlage des Materialflussmodells bilden Prozesskettenanalysen, wie sie auch in der Ökobilanz (Life Cycle Assessment; LCA) genutzt werden. Analog zu Ökobilanzen wird die Nutzung forstlicher Ressourcen auch im vorliegenden Modell über den gesamten Lebensweg betrachtet, d.h. von der Wiege (Ressourcenentnahme) bis zur Bahre (Entsorgungsprozess) [16].

Der gewählte Ansatz unterscheidet sich zum einen von traditionellen Materialflussmodellen, die im Allgemeinen ebenso auf die Darstellung des Materialflusses auf Prozessebene verzichten wie auch auf die Modellierung von Vorketten [17]. Zum anderen, in Ergänzung zu einer traditionellen Ökobilanz, werden die konkurrierenden und kaskadischen Nutzungsmöglichkeiten von $\mathrm{Holz}$ auf allen Prozessstufen miteinander verknüpft bzw. simultan betrachtet und bei der abschließenden Bilanzierung der ökologischen Wirkungen des Referenzsystems und des Zukunftsszenarios berücksichtigt. Dieses Vorgehen gestattet die Analyse und Bewertung von einzelnen Produkten und Prozessketten unter Berücksichtigung der gesamten Produktpalette der Holzindustrie. Hierdurch können potenzielle Rückkopplungseffekte erfasst, aber auch die branchenweiten Substitutionseffekte und Nachfrageänderungen identifiziert und quantifiziert werden. Der verstärkte Einsatz von Leichtbauplatten verändert bspw. das Angebot an Industrierestholz mit möglichen Implikationen für die nachnutzenden Prozesse im Sinne einer veränderten Verfügbarkeit für die stoffliche Nutzung oder eines zusätzlichen Bedarfs an fossilen Brennstoffen.

Der gewählte Ansatz stellt eine Erweiterung der traditionellen Materialflussanalyse [18] um Methodenbestandteile der Ökobilanz dar. In der vorliegenden Analyse werden konkret das Konzept der Sachbilanz sowie Methoden der Wirkungsabschätzung von Emissionen und 

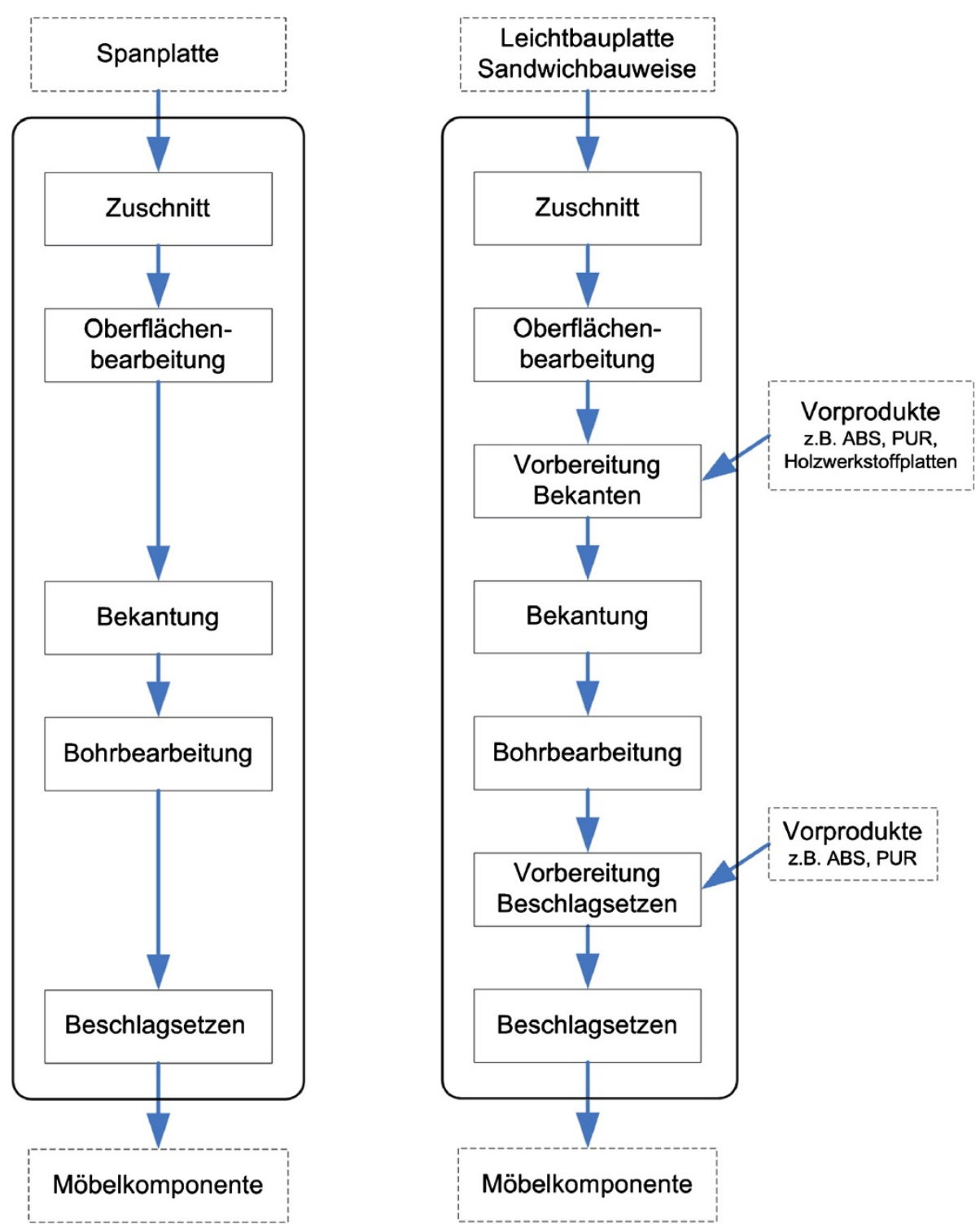

Abbildung 3 Prozessschritte der Möbelfertigung aus Spanplatten (linke Abbildung) und aus Leichtbauplatten (rechte Abbildung).

Ressourcenentnahmen in das Materialflussmodell implementiert. Während die traditionelle Materialflussanalyse im Allgemeinen auf den Stoffstrom eines Materials (oder mehrerer Materialien) fokussiert, ohne einen expliziten Bezug auf die weiteren, in den Produktionsprozessen ebenfalls genutzten Materialien und Energieträger zu nehmen, konzentriert sich die Ökobilanz auf ein Produkt-bezogenes System mit seinen vorgelagerten und (u.U.) nachgelagerten Prozessketten. Die Berücksichtigung konkurrierender Produkte ist der Ökobilanz im Allgemeinen wesensfremd. Dahingegen ist in der Materialflussanalyse die Nutzung von Wirkungsabschätzungsmethoden zur Bewertung von Umweltwirkungen, wie sie fester Bestandteil der Ökobilanz ist, eher unüblich.
Aus Sicht der Materialflussanalyse bedeutet die gewählte Vorgehensweise eine produktions- und produktbezogene Fundierung des jeweils dargestellten Materialflusses bzw. -flüsse [19]. Aus Sicht der Ökobilanz könnte der gewählte Ansatz als umfassende Systemerweiterung einer traditionellen Ökobilanz verstanden werden. Er eröffnet die Möglichkeit, auch komplexe Systemvergleiche aufgrund von Materialsubstitution durch bspw. Innovationen und damit verbundene Veränderungen der Nutzungspfade und -mengen hinsichtlich ihrer ökologischen Wirkungen innerhalb eines bestehenden Systems zu bewerten. Aufgrund der Zielstellung der vorliegenden Analyse ist die Definierung einer funktionellen Einheit nicht zweckdienlich, was aber nicht heißt, dass man in anderen Kontexten eine solche nicht definieren könnte [20]. 
Die betrachteten treibhauswirksame Gase (i.F. THG) Kohlenstoffdioxid $\left(\mathrm{CO}_{2}\right)$, Methan $\left(\mathrm{CH}_{4}\right)$ und Distickstoffmonoxid $\left(\mathrm{N}_{2} \mathrm{O}\right.$, Lachgas) sind wesentliche Emissionen bei der Nutzung biogener Ressourcen allgemein. Schwefelhexafluorid $\left(\mathrm{SF}_{6}\right)$ wird aufgrund seiner besonders hohen Wirkungsweise im Bereich der GWP mit in die Berechnungen eingezogen. Die weiteren Emissionsstoffe - flüchtige organische Verbindungen ohne Methan (NMVOC) und Formaldehyd $\left(\mathrm{CH}_{2} \mathrm{O}\right)$ - werden aufgrund der respiratorischen Effekte, d.h. der Wirkungen auf die menschlichen Gesundheit, ausgewiesen.

Die Emissionen der einzelnen Emissionsstoffe werden entsprechend der Vorgehensweise des IPCC [21] in Kohlenstoffdioxid-Äquivalente mit einem Zeithorizont von 100 Jahren sowie entsprechend dem Vorgehen zur Berechnung des Ozonbildungspotenzials der Wirkungsabschätzungsmethode Impact2002+ [22] in Ethylen-Äquivalente umgerechnet (s. Tabelle 2). Dies ermöglicht einen Vergleich der einzelnen Emissionsstoffe hinsichtlich des jeweiligen Treibhauspotenzials (engl.: global warming potential; i.F. GWP) sowie hinsichtlich der respiratorischen Effekte. Die Untersuchung dieser Wirkungen (GWP und menschliche Gesundheit) wird im Hinblick auf die aktuelle politische Diskussion und auf die Fachgespräche innerhalb der Holzindustrie als die wesentlichen beiden angesehen und daher wird von der Untersuchung weitere Kategorien abgesehen.

\section{Referenzsystem}

Das für die Analyse relevante Referenzsystem umfasst die gesamte holzbe- und holzverarbeitende Industrie in Deutschland einschließlich der Papierindustrie [23]. Eine vollständige Abbildung der Umweltwirkungen erfordert auch die Berücksichtigung der relevanten vorund nachgelagerten Sektoren, wie die Bereitstellung von Vorprodukten durch die chemische Industrie oder die abschließende thermische Behandlung von Holz (s. Abbildung 4). Bei holzbasierten Rest- und Abfallstoffen wird die weitere stoffliche oder energetische Verwertung explizit erfasst. Die gewonnene Energie wird in der

Tabelle 2 Koeffizienten der Kohlenstoffdioxid- bzw. Ethylen-Äquivalente

\begin{tabular}{lrl}
\hline Emissionsstoff & $\begin{array}{l}\mathrm{GWP} \text { in } \mathrm{CO}_{2} \text {-Äquivalenten } \\
{[21]}\end{array}$ & $\begin{array}{l}\text { Respiratorische Effekte in } \\
\text { Ethylen-Äquivalenten [22] }\end{array}$ \\
\hline $\mathrm{CO}_{2}$ & 1 & \\
$\mathrm{CH}_{\mathbf{4}}$ & 25 & \\
$\mathrm{~N}_{\mathbf{2}} \mathrm{O}$ & 298 & \\
$\mathrm{SF}_{6}$ & 22.800 & 0,501 \\
Formaldehyd & & 0,303 \\
NMVOC & & \\
\hline
\end{tabular}

Energieversorgung genutzt (s. Abbildung 4). Die Ausnahme bildet die Forstwirtschaft, da Holzernteprozesse nicht betrachtet werden.

Nach Lohmann [11] wird die Holzindustrie untergliedert in die Wertschöpfung am oder nahe am Rundholz bzw. Rohholz, genannt die holzbearbeitende Industrie, und das produzierende Gewerbe, genannt die holzverarbeitende Industrie, welche Vorprodukte $\mathrm{zu}$ Endprodukten verarbeitet.

Die holzbearbeitende Industrie umfasst die Prozessketten von der Nutzung der forstlichen Ressourcen bis zum (Zwischen-)Produkt wie Holzwerkstoffplatten, Schnittholz oder Papier [24]. Hierzu zählt auch die Herstellung der Leichtbauplatten. Die forstlichen Ressourcen, gegliedert nach Holzartengruppen und -sortimenten, werden von der Forstwirtschaft bereitgestellt, im System aktuell aber nur durch Transportprozesse vom Wald zum Werk berücksichtigt (s. Abbildung 4).

Die holzverarbeitende Industrie fertigt aus den Waren der Holzbearbeitung vielfältige Zwischen- und Endprodukte (s. Abbildung 4) [25]. Hier werden die Leichtbauplatten zu Endprodukten (Möbel) weiter verarbeitet. Die Verknüpfung zwischen Holzbe- und -verarbeitung erfolgt über Transportprozesse, die entsprechend Zimmer et al. [26] abgebildet werden.

Die Produkte werden am Ende ihrer Nutzungsphase entweder rezykliert oder der thermischen Verwertung zugeführt.

Bei allen modellierten Prozessen werden neben dem holzbasierten Vorprodukt auch die Herstellung sowie Gewinnung relevanter nicht-holzbasierter Inputs (bspw. Klebstoffe) erfasst.

Das Gesamtsystem ist nachfrageorientiert. Das heißt, die Nachfrage nach Zwischen- und Vorprodukten wird, unter Berücksichtigung technischer Restriktionen, durch die Nachfrage nach Endprodukten wie bspw. Möbel determiniert.

Das Modell bildet Materialflüsse als Prozessketten von der Ressourcenentnahme über Zwischenprodukte bis $\mathrm{zu}$ den Endprodukten ab. Gekoppelt an die Materialflüsse werden treibhauswirksame luftgetragene Emissionen betrachtet, die während der Produktions- und Transportprozesse freigesetzt werden.

Räumliche Systemgrenze ist die administrative Grenze Deutschlands. Betrachtet wird die hier ansässige Holzindustrie sowie den Rohstoff Holz nutzende Industrien (s. Abbildung 4). Das Referenzjahr ist das Jahr 2005.

Neben Informationen zur Bereitstellung forstlicher Ressourcen werden auch für weitere Produkte und Prozesse, wie z.B. für notwendige Rohstoffe oder Vorprodukte, ökologische Kennzahlen als generische (allgemeingültige) Informationen aus Datenbanken entnommen. In gleicher Weise werden Transporte bzw. 


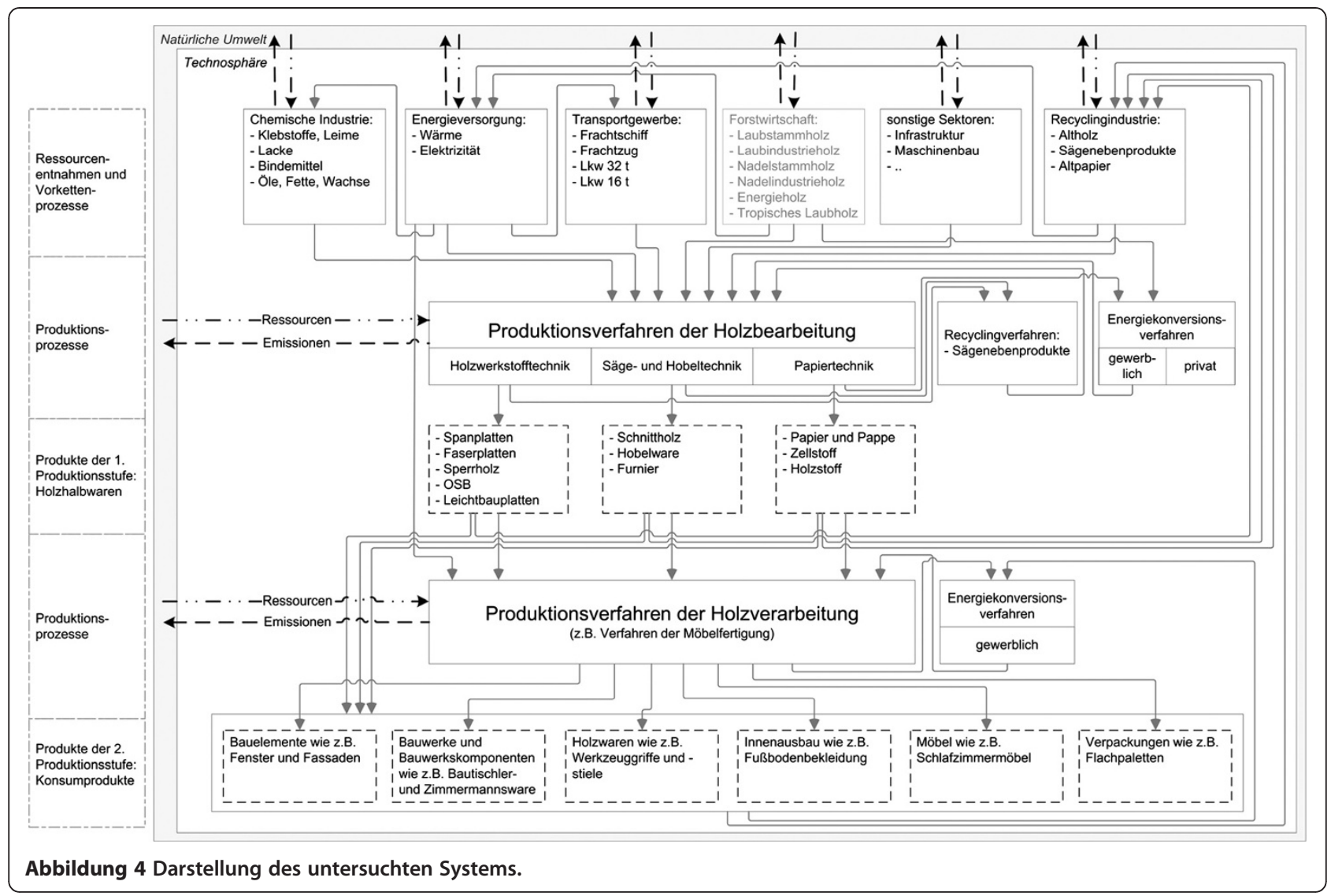

Transportmittel und die Energiebereitstellung abgebildet. Genutzt werden hierzu Daten aus der Datenbank ecoinvent $[27,28]$, die soweit notwendig an die Bedingungen des Modells angepasst wurden. Des Weiteren wurden für die Modellierung Informationen und Kennzahlen über eine Literaturrecherche ermittelt [5,29]. Ergänzend wurden Informationen von Firmen eingeholt sowie Berechnungen und Annahmen bspw. auf Grundlage vergangener Jahre getroffen.

Die Modellierung des Systems erfolgt mit der Software umberto [30].

\section{Zukunftsszenario}

Zur Quantifizierung der Folgen einer Marktdurchdringung von Leichtbauplatten auf THG-, NMVOCund Formaldehyd-Emissionen muss zunächst der technisch mögliche Marktanteil von Leichtbauplatten in der Möbelindustrie ermittelt werden.

Für die Bestimmung des technisch maximal möglichen Marktanteils von Sandwichplatten wurde die Spanplattenmenge abgeschätzt, die - unter Berücksichtigung konstruktiver Limitierungen - durch leichte Holzwerkstoffplatten ersetzt werden kann (s. Tabelle 3). Diese Berechnung wurde auf Basis der Produktionsmenge verschiedener Möbelarten [31] - Stand 2005 - und durch eigene Abschätzung des jeweilig substituierbaren Anteils und der durchschnittlichen Fläche pro Möbelart berechnet.

Die ermittelte Substitutionsmenge von 90.685,9 Tsd. $\mathrm{m}^{2}$ wurde in $\mathrm{m}^{3}$ umgerechnet, wobei eine durchschnittliche Dicke von $28 \mathrm{~mm}$ angenommen wird, um mit Leichtbauplatten realisierte, im Vergleich zu Spanplatten größere Plattendicken in Möbeln abzubilden. So ergeben sich $2.539 * 10^{3} \mathrm{~m}^{3}$ Spanplattenmaterial, welches durch Leichtbauplatten substituiert werden kann. Mit der gesamtdeutschen Spanplattenproduktion im Jahr 2005 verglichen [32], entspricht dieser einem Anteil von $27 \%$.

Für die Analyse wird daher angenommen, dass die Produktion der leichten Platten entsprechend des ermittelten Potenzials von $27 \%$ anteilig die Produktion der Spanplatten ersetzen kann. Hierdurch wird die am Markt nachgefragte Plattenmaterialmenge abgebildet.

\section{Ergebnisse}

Die partielle Substitution von Spanplatten in der Möbelindustrie durch Wabenplatten führt $\mathrm{zu}$ einer durchgängigen Minderung der untersuchten Emissionen im Gesamtsystem (s. Tabelle 4). Hinsichtlich des globalen Erwärmungspotenzials beträgt der Rückgang knapp 1 Mio.t $\mathrm{CO}_{2}$ - ̈̈quivalente, bei den respiratorischen Effekten 116 t Ethylen-Äquivalente. 
Tabelle 3 Abschätzung der substituierbaren Menge an Spanplatten, strukturiert nach Möbeltypen

\begin{tabular}{|c|c|c|c|c|}
\hline Möbeltyp & Produktion [1.000 St.] [29] & $\begin{array}{l}\text { Substitutionsanteil [\%] } \\
\text { (eigene Abschätzungen) }\end{array}$ & $\begin{array}{l}\text { Fläche pro Stück }\left[\mathrm{m}^{2}\right] \\
\text { (eigene Berechnungen) }\end{array}$ & $\begin{array}{l}\text { Substitutionsmenge } \\
{\left[1.000 \mathrm{~m}^{2}\right]}\end{array}$ \\
\hline Küchenarbeitsplatten & 24.809 & 70 & 0,36 & $6.251,9$ \\
\hline Betten & 1.935 & 50 & 3,40 & $3.289,5$ \\
\hline Schränke & 5.112 & 70 & 14,36 & $51.385,8$ \\
\hline andere Möbel für Schlafzimmer & 7.659 & 80 & 1,04 & $6.372,3$ \\
\hline Wohnzimmerschränke & 792 & 80 & 5,91 & $3.744,6$ \\
\hline Regalwände & 3.549 & 80 & 5,91 & $16.779,7$ \\
\hline andere Möbel für Wohnzimmer & 3.440 & 80 & 1,04 & $2.862,1$ \\
\hline Summe Plattenmaterial $\left[1.000 \mathrm{~m}^{2}\right]$ & & & & $90.685,9$ \\
\hline
\end{tabular}

Beim GWP dominiert mit knapp 80\% Anteil an dem gesamten Minderungspotenzial Kohlenstoffdioxid den Reduktionsbeitrag durch Leichtbauplatten. Der Anteil von Lachgas beträgt dahingegen nur gut 15\%; Methan trägt knapp 5\% zum Minderungspotenzial bei. Der Anteil von Schwefelhexafluorid ist vernachlässigbar gering.

Die Holzbearbeitung trägt insgesamt mit $56 \% \mathrm{zu}$ dem reduzierten Emissionsvolumen bei; die Holzverarbeitung mit 44\%. Auf der Ebene der einzelnen Schadstoffe ist ein abweichendes Muster festzustellen. Während bei Methan beide Prozessstufen nahezu paritätisch zur Emissionsminderung beitragen, liegt der Anteil der Holzbearbeitung an dem Emissionsminderungspotenzial von Kohlenstoffdioxid bei knapp $66 \%$ und von Schwefelhexafluorid bei gut $60 \%$. Dahingegen trägt bei Lachgas die Holzbearbeitung mit weniger als 2\% nur geringfügig zur Emissionsminderung bei.

Bei den Emissionen mit respiratorischem Effekt, d.h. die Wirkungen auf die menschliche Gesundheit, hat NMVOC einen Anteil von etwa 56\% am Minderungspotenzial; Formaldehyd trägt mit $44 \%$ bei. Relevant für das Reduktionspotenzial sind nur die durch die Holzbearbeitung verursachten Emissionen; der Beitrag der Holzverarbeitung ist absolut und relativ betrachtet vernachlässigbar gering. Anzumerken bleibt, dass die Emissionen von Formaldehyd in der holzverarbeitenden Industrie zunimmt; die Zunahme aber ebenfalls vernachlässigbar ist (s. Tabelle 5).

Tabelle 4 Veränderung der Treibhausgasemissionen in der Holzindustrie im Vergleich zum Referenzsystem; in t Kohlenstoffdioxid-Äquivalente pro Jahr

\begin{tabular}{lrrr}
\hline Emissionen & Holzbearbeitung & Holzverarbeitung & Summe \\
\hline $\mathrm{CO}_{\mathbf{2}}$ & -509.465 & -255.609 & -765.074 \\
$\mathrm{CH}_{\mathbf{4}}$ & -24.146 & -23.298 & -47.443 \\
$\mathbf{N}_{\mathbf{2}} \mathbf{O}$ & -2.469 & -145.730 & -148.200 \\
$\mathrm{SF}_{\mathbf{6}}$ & -81 & -53 & -134 \\
Summe & -536.161 & -424.691 & -960.851 \\
\hline
\end{tabular}

Innerhalb der Holzbearbeitung sind die Ursachen für das Minderungspotenzial bei Treibhausgasen eindeutig zuzuordnen (s. Abbildung 5), wobei das Potenzial nicht in einem singulären Prozessschritt $\mathrm{zu}$ finden, sondern auch durch die Betrachtung der Vorketten bedingt ist. Drei wesentliche Gründe lassen sich für die Minderung der Emissionen identifizieren: Erstens erfordert die Leichtbauplattenproduktion einen geringeren Einsatz an Kleb- und Bindemitteln. Der verminderte Bedarf an Klebstoffen und Bindemitteln bei den leichten Platten induziert eine verringerte Produktion bei diesen, so dass die Emissionen deutlich sinken. Die Relevanz von Klebstoffen und Bindemitteln variiert je nach Schadstoff zwischen 55\% (Lachgas) und 80\% (Methan). Zweitens sinkt durch die volumenlimitierte Beladung der LKWs bei Leichtbauplatten im Gegensatz zur gewichtslimitierten Beladung bei Spanplatten der LKWbasierte Transportaufwand und es kommt $\mathrm{zu}$ einem nennenswerten Beitrag zum Minderungspotenzial: je nach Schadstoff beträgt der Anteil der LKW-gestützten Transportprozesse zwischen 5\% (Methan) und 15\% (Lachgas). Als dritte Quelle für das Minderungspotenzial ist die thermische Nutzung in der Entsorgungsphase $\mathrm{zu}$ nennen. Leichtbauplatten weisen einen erheblich niedrigeren Energiebedarf gegenüber konventionellen Spanplatten auf. Die Relevanz hierbei wird bei Kohlenstoffdioxid (Anteil: knapp 20\%) und bei Lachgas (Anteil: 25\%) besonders deutlich. Jegliches fossiles Kohlenstoffdioxid wird hier vollständig berücksichtigt und ausgewertet; biogenes Kohlenstoffdioxid nicht, da

Tabelle 5 Die respiratorischen Effekte der Holzindustrie im Vergleich zum Referenzsystem; in t EthylenÄquivalente pro Jahr

\begin{tabular}{lrrr}
\hline Emissionen & Holzbearbeitung & Holzverarbeitung & Summe \\
\hline NMVOC & $-61,10$ & $-0,03$ & $-61,13$ \\
Formaldehyd & $-48,20$ & 0,01 & $-48,30$ \\
Summe & $-109,30$ & $-0,04$ & $-109,34$ \\
\hline
\end{tabular}




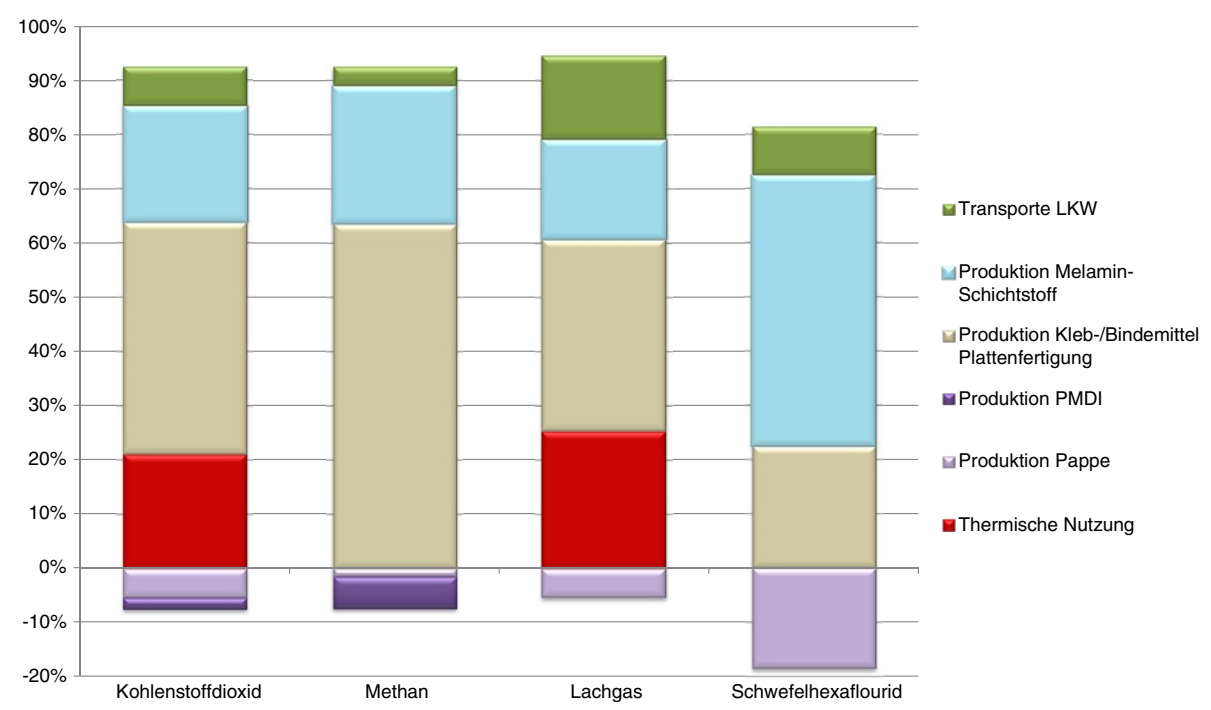

Abbildung 5 Treibhauswirksame Emissionen der holzbearbeitenden Industrie; Quellen mit einem Beitrag kleiner 2\% sind nicht dargestellt (Anm.: Die einzelnen Säulen geben die Aufteilung der einzelnen Emissionsquellen in Prozent an. Eine Säule erreicht nur dann den Wert von $100 \%$, wenn alle Emissionsquellen treibhausgasmindernd wirken).

auch der Forst als Senke z. Zt. nicht berechnet wird und die Betrachtung bei einem anderen Vorgehen inkonsistent wird.

Es lassen sich zwei Gründe identifizieren, die das mögliche Minderungspotenzial mindern, d.h. zusätzliche Emissionen freigesetzt werden. Der Einsatz einer Papiermittellage bewirkt höhere Emissionen in der Papierproduktion und ebenso der steigende Bedarf an Polymerem Diphenylmethandiisocyanat (PMDI), einem Bindemittel der Holzwerkstoffindustrie. Letzteres vermindert insbesondere bei Methan das Minderungspotenzial; der Anteil beträgt knapp 10\%. Ersteres wirkt insbesondere auf die Emissionen von Schwefelhexafluorid und Lachgas sowie Kohlenstoffdioxid - mit abnehmender Bedeutung.

Ein vergleichbares Bild zeigt sich für die Emission von NMVOC (s. Abbildung 6). Im Gegensatz dazu ist die Quelle der Formaldehydemission nahezu vollständig die Produktion der Klebstoffe und Bindemittel für die Plattenfertigung.

Im Gegensatz zur holzbearbeitenden Industrie können für die holzverarbeitende Industrie keine eindeutigen Muster für die Quellen des Minderungspotenzials identifiziert werden. Dies liegt im Wesentlichen an der Vielfalt der Produktionsprozesse und Prozessschritte in der Möbelfertigung.

\section{Diskussion}

Insgesamt führt der verstärkte Einsatz von Leichtbauplatten zu einer Verminderung der treibhauwirksamen Emissionen um 1 Mio.t $\mathrm{CO}_{2}$ - ̈̈quivalente und zu einer Verminderung der respiratorisch wirksamen Emissionen um $110 \mathrm{t}$ Ethylen-Äquivalente (s. hierzu Kapitel 4). Im Vergleich zu den gesamten, von dem System freigesetzten Emissionen sind die Minderungen als gering zu bezeichnen: sie liegen für alle betrachteten Emissionen im kleinen einstelligen Prozentbereich; z.B. für Kohlenstoffdioxid bei 2,4\%, für NMVOC bei lediglich 0,7\%.

Das ermittelte Emissionsminderungspotenzial beschreibt hierbei das technisch machbare Potenzial und somit die maximal möglichen Emissionsminderungen unter Berücksichtigung der heute bekannten Verfahren bzw. deren technische Effizienz bei der Herstellung von Leichtbauwerkstoffen.

Tatsächlich sind mit einer zunehmenden Verbreitung von Leichtbauplatten einerseits Skalenerträge zu erwarten, die aber nicht abschätzbar sind. Andererseits basieren die Werte auf Abschätzungen, die zwar als verlässlich anzusehen sind, die aber nur bedingt durch Erfahrungen aus der Industrie falsifiziert werden konnten. Das heißt, möglicherweise ist die unterstellte Produktionseffizienz $\mathrm{zu}$ optimistisch und die oben angesprochenen Skalenerträge wurden im Modell schon vorweggenommen.

Jedoch wird das tatsächlich zu erwartende Reduktionspotenzial auch wesentlich durch ökonomische Randbedingungen bestimmt. Inwieweit und wann die modellierte Veränderung des Holzwerkstoffplattenmarktes eintreten wird, hängt von zahlreichen Faktoren ab: Eine entscheidende Einflussgröße ist der Holzpreis als Treibkraft für eine ressourceneffizientere Holznutzung. Darüber hinaus sind der Rohölpreis als Kostentreiber für Energie- und Klebstoffkosten sowie Entwicklungen in der Möbelfertigung und Modetrends bei der Endkundennachfrage von nennenswerter Relevanz. Eine Aussage über die tatsächliche Entwicklung des Absatzes leichter Platten 


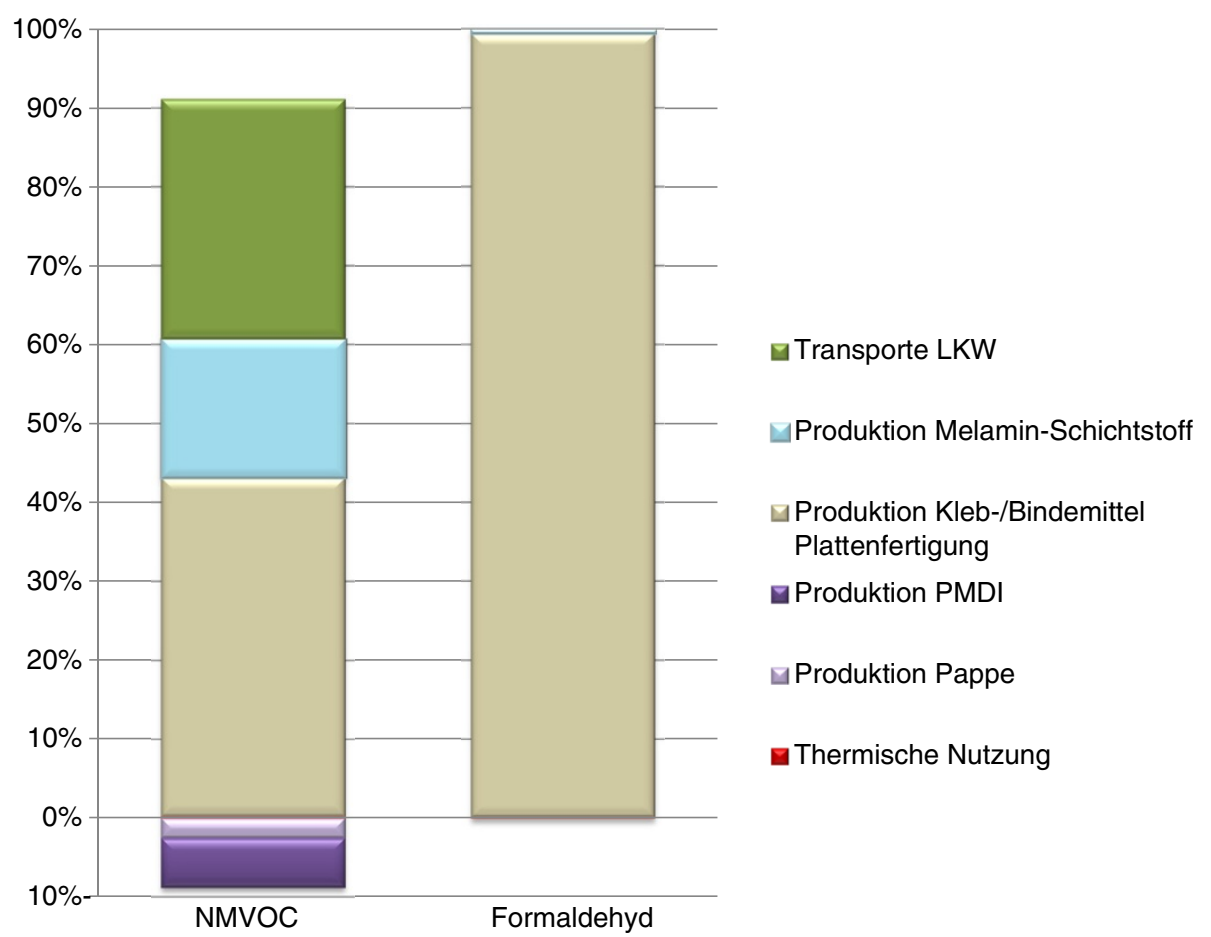

Abbildung 6 Respiratorisch wirksame Emissionen der holzbearbeitenden Industrie.

und damit über das ökonomische Emissionsminderungspotenzial erfordern weitere Untersuchungen und mithin eine ökonomische Erweiterung des gewählten Ansatzes. Aus Erfahrung werden technische Minderungspotenziale aus den o.g. Gründen selten vollständig ausgeschöpft. Das tatsächliche Emissionsminderungspotenzial wird eher geringer ausfallen. Eine genaue Abschätzung liegt aber außerhalb des Fokus dieser Arbeit und des vorliegenden Modells.

Die technische Umsetzbarkeit einer "leichten“ Konstruktion im Produktionsprozess mit hoher Prozesssicherheit sowie die Auswirkungen im Hinblick auf das Design von Möbeln wurden im Rahmen dieser Analyse ebenfalls nicht betrachtet. Ein möglicher Verzicht der holzverarbeitenden Industrie auf eine Nachfrage nach leichten Holzwerkstoffplatten aus prozesstechnischen Überlegungen würde ebenfalls das mögliche Emissionsminderungspotenzial mindern.

Auf der anderen Seite wurde von anderen Bauformen sowie Einsatzmöglichkeiten von Leichtbauplatten abstrahiert, die das Emissionsminderungspotenzial erweitern könnten.

Die Analyse verdeutlicht, dass nicht benötigte Klebstoffe und Bindemittel einen deutlichen Anteil zum Einsparpotenzial beitragen. Diese Betriebsstoffe sind sowohl ressourcen- als auch energieintensiv im Herstellungsprozess und setzen somit vergleichsweise große Mengen THG frei. Dieses Ergebnis, d.h. die Relevanz der Bindemittel, lässt sich auf alle Bauformen von leichten Platten mit Spanplattendeckschichten und Papiermittellage übertragen. Die Dichte der Papiermittellage, d.h. deren Gestaltung, ist demnach von untergeordneter Bedeutung.

Auch wenn der Rückgang der Klebstoff- und Bindemittelmengen den Rückgang des globalen Erwärmungspotenzials dominiert, so ist ebenfalls das Einsparungspotenzial im Bereich der Logistik nicht zu vernachlässigen. Gleiches gilt für die Emissionen von NMVOC im Bereich der respiratorischen Effekte.

Während die obigen Effekte insgesamt zu erwarten waren, so gilt dies nicht für das Einsparpotenzial bei der thermischen Nutzung von Holz bei einer erweiterten Systembetrachtung. Wie oben schon angesprochen, verändert der verstärkte Einsatz von Leichtbauplatten das inputseitige Angebot an Industrierestholz, das stofflich und energetisch genutzt werden kann, was in der vorliegenden Ebene der Untersuchung zu einer verminderten Holznachfrage führt. Outputseitig kann durch die Substitution von Spanplatten durch Sandwichplatten weniger Spanplattenmaterial thermisch verwertet werden. Dieser Rückgang wird nur marginal durch eine vermehrte Verfügbarkeit von Leichtbauplatten kompensiert, die noch dazu einen geringen Energiegehalt haben als Spanplatten. Die „fehlenden "Energiemengen müssen durch andere Energieträger mit potenziell höheren spezifischen Emissionswerten ersetzt 
werden. Die Ergebnisse zeigen aber, dass umweltrelevante Substitutionseffekte die Vorteile aus der Leichtbauplattenproduktion in dem betrachteten System nicht aufwiegen können.

\section{Fazit und Ausblick}

Unter den genannten Rahmenbedingungen betrachtet, kann der Einsatz von Sandwichplatten mit Papiermittellage eine Perspektive bieten, die betrachteten Luftemissionen der Holzindustrie zu senken, wobei die zu erreichenden Gewinne im kleinen einstelligen Prozentsatz liegen; z.B. bei Kohlenstoffdioxid bei 2,4\%. Die größten Einsparungen können im Bereich der Produktion von Klebstoffen und Bindemitteln erreicht werden. Für die verschiedenen Bauformen leichter Platten aus Spanplattendeckschichten mit Papiermittellage sind die Klebstoffe von zentraler Bedeutung; die Ausführung der einzelnen Komponenten einer solchen Sandwichplatte ist zweitrangig.

Die gewählte Vorgehensweise ermöglicht ein tiefer gehendes Verständnis der komplexen horizontalen und vertikalen Vernetzungen innerhalb der Holzindustrie, da es konkurrierende und kaskadische Beziehungen zwischen den Prozessen auf unterschiedlichen Prozessstufen erfasst. Hierdurch können Rückkopplungseffekte ebenso berücksichtigt werden wie branchenweite Substitutionsmöglichkeiten. Wie im Rahmen dieser Arbeit gezeigt wurde, ermöglicht der Ansatz auch komplexe Systemvergleiche aufgrund von Materialsubstitution - hier: partielle Substitution der Spanplatte durch Leichtbauplatten in der Möbelindustrie - und damit verbundene Veränderungen der Nutzungspfade und -mengen hinsichtlich ihrer ökologischen Wirkungen innerhalb der Holzindustrie zu bewerten. Der gewählte Ansatz hebt sich damit von der traditionellen Materialflussanalyse ab.

Die umweltrelevanten Konsequenzen eines verminderten stofflichen Angebots an Spanplattenmaterial auf die Forstwirtschaft können derzeit nicht weiter betrachtet werden, da die Forstwirtschaft noch nicht erfasst wird. Daher ist es von wesentlichem Interesse, die deutsche Forstwirtschaft als Produzent des Rohstoffs Holz in der Modellierung abzubilden und die Analysen so zu vervollständigen. Weitere Arbeiten ergeben sich in der Analyse der ökonomischen Wirkungen der Substitutionseffekte. Die Software umberto wurde unter anderem ausgewählt mit dem Ziel, dass das Modell in der Lage ist, neben ökologischen auch ökonomische Kennzahlen mitzuführen und zu berechnen.

\section{Competing interests}

The authors declare that they have no competing interests.

\section{Authors' contributions}

SF carried out the model, calculated baseline and scenario and wrote main parts of this article. WRP wrote parts of the article and supported intensively the development of the model and of the scenarios. LS supported the writing of the article comprehensively. All authors read and approved the final manuscript.

\section{Author details}

${ }^{1}$ Karlsruher Institut für Technologie, Institut für Technikfolgenabschätzung und Systemanalyse, Hermann-von-Helmholtz-Platz 1, D-76344 Eggenstein-Leopoldshafen, Germany. ${ }^{2}$ Technische Universität Darmstadt, Institut IWAR, Fachgebiet Industrielle Stoffkreisläufe, Petersenstr 13, D-64287 Darmstadt, Germany.

Received: 30 July 2012 Accepted: 14 December 2012

Published: 1 March 2013

\section{References}

1. Anonym: Leichtbau - dauerhafter Trend oder kurze Mode? HolzZentralblatt 2009, 23:575.

2. Hülsbusch B, Falk W, Haasler M, Steib W: Der schlafende Riese. HK Special Holzwerkstoffe 2010, 6:64-67.

3. Stosch M: BM Special: Leichtbau - Werkstoffe Technologie Verarbeitung. Leinfelden-Echterdingen: Konradin Verlag; 2009.

4. Anonym: Leichte Möbel rationeller herstellen. Holz-Zentralblatt 2009, 3:61.

5. Poppensieker J, Thömen H: Wabenplatten für den Möbelbau. Hamburg: Bundesforschungsanstalt für Forst- und Holzwirtschaft; 2005.

6. Feifel S, Faul A, Schebek L: Vergleichende ökologische Analyse leichter Holzwerkstoffplatten mit unterschiedlichem Aufbau. Holztechnologie 2011, 52:3.

7. Institut Bauen und Umwelt e.V. (Hrsg.): Umwelt-Produktdeklaration nach ISO 14025 - Egger Eurolight ${ }^{\circledR}$ - Rohe und beschichtete Leichtbauplatten. Königswinter; 2008.

8. Shalbafan A, Luedtke A, Welling J, Thoemen H: Comparison of foam core materials in innovative lightweight wood-based panels. Eur Wood Prod 2012, 70:287-292. doi:10.1007/s00107-011-0552-0.

9. Kharazipour A, Ritter N, von Werder H-K, Bohn C: Entwicklung leichter dreischichtiger Spanplatten auf Basis nachwachsender Rohstoffe. Holztechnologie 2011, 52:5.

10. Dix B, Meinlschmidt $P$, van de Flierdt $A$, Thole V: Leichte Spanplatten für den Möbelausbau aus Rückständen der landwirtschaftlichen Produktion. Teil 1: Verfügbarkeit der Rohstoffe. Holztechnologie 2009, 50:2.

11. Lohmann U (Hrsg.): Holz-Lexikon 5. Auflage. Leinfelden-Echterdingen. DRW; 2003.

12. Hischier R, Althaus H-J, Werner F: Developments in Wood and Packaging Materials Life Cycle Inventories in ecoinvent. Int J Life Cycle Assess 2005, 10:50-58. doi:10.1065/lca2004.11.181.6.

13. Rehau: Stützkanten-Technologie. http://www.rehau.com/DE_de/ design_fuer_moebel/.

14. Hettich: Leichtbaulösungen. [http://www.hettich.com/de_DE/startseite.html]

15. Häfele: Intelligente Möbel mit innovativer Beschlagtechnik von Häfele. Smart Spaces - Räume mit Mehrwert [http://www.haefele.de/]

16. Klöpffer W, Grahl B: Ökobilanz (LCA) - Ein Leitfaden für Ausbildung und Beruf. Weinheim: Wiley-VCH; 2009.

17. Mantau U, Bilitewski B: Stoffstrom-Modell-Holz. Bestimmung des Aufkommens, der Verbreitung und des Verbleibs von Holzprodukten. Verband Deutscher Papierfabriken e.V. 2005.

18. Baccini P, Bader H-P: Regionaler Stoffhaushalt - Erfassung, Bewertung und Steuerung. Heidelberg * Berlin * Oxford: Spektrum Akademischer Verlag; 1996.

19. Uihlein A, Poganietz WR, Schebek L: Carbon flows and carbon use in the German anthroposphere: An inventory. Res Con Rec 2006, 46:410-429. doi:10.1016/j.resconrec.2005.09.001.

20. Sandén BA, Karlström M: Positive and negative feedback in consequential life-cycle assessment. J Cle Prod 2007, 15:1469-1481. doi:10.1016/j. jclepro.2006.03.005.

21. Forster $P$, Ramaswamy $V$, et al: Changes in Atmospheric Constituents and in Radiative Forcing. In Climate Change 2007: The Physical Science Basis. Cambridge/New York: Cambridge University Press: Contribution of Working Group I to the Fourth Assessment Report of the Intergovernmental Panel on Climate Change; 2007.

22. Jolliet O, Margni M, et al: IMPACT 2002+ - A Life Cycle Impact Assessment Methodology - Version 2.1; Characterization Factors. Ecole Polytechnique Fédérale de Lausanne; 2005.

23. Forest-Based Sector Technology platform: Innovative and Sustainable Use of Forest Resources - Vision 2030. 2030 [www.forestplatform.org]

24. Westphal P: Modell der Stoffströme der Holzbearbeitenden Industrie in Deutschland. Hochschule für angewandte Wissenschaft und Kunst Hildesheim: Diploma Thesis; 2007. 
25. Brommer E: Stoffstrommodell der Holzverarbeitenden Industrie in Deutschland. FH Trier, Umwelt-Campus Birkenfeld: Diploma Thesis; 2008.

26. Zimmer B, Wegener G, Nebel B: Analyse der Transportketten von Holz, Holzwerkstoffen und Restholzsortimenten als Grundlage für produktbezogene Ökobilanzen. München/Kuchl: Spektrum-Verlag; 2004.

27. Swiss Centre for Life Cycle Inventories: Ecoinvent data. Empa, St. Gallen, Switzerland: Ecoinvent Centre; 2005

28. Frischknecht $\mathrm{R}$, Jungbluth $\mathrm{N}$, et al: The ecoinvent database: overview and methodological framework. Int I Life Cycle Assess 2004, 10:3-9. doi:10.1065/ Ica2004.10.181.1.

29. Bitter W: Forst und Holz Marktbilanz 2007. Bonn: Zentrale Markt- und Preisberichtstelle; 2007.

30. Institut für Umweltinformatik: umberto 5.5. Hamburg; 2009 .

31. Holzmann Verlag (Hrsg.): Möbel, Zahlen, Daten. Hamburg: Holzmann-Verlag; 2006.

32. Statistisches Bundesamt: Produzierendes Gewerbe - Produktion im Produzierenden Gewerbe - Jahr 2007. Fachserie 4 - Reihe 3.1. Wiesbaden; 2008.

doi:10.1186/2190-4715-25-5

Cite this article as: Feifel et al:: The utilization of light weight boards for reducing air emissions by the German wood industry - a perspective?

Environmental Sciences Europe 2013 25:5.

\section{Submit your manuscript to a SpringerOpen ${ }^{\circ}$} journal and benefit from:

- Convenient online submission

- Rigorous peer review

- Immediate publication on acceptance

- Open access: articles freely available online

- High visibility within the field

- Retaining the copyright to your article 